\title{
Experimental studies of rapid sperm transport in rabbits
}

\author{
J. W. Overstreet and R. A. Tom
}

Department of Human Anatomy and Department of Obstetrics and Gynecology, School of Medicine, University of California, Davis, California 95616, U.S.A.

\begin{abstract}
Summary. When non-motile spermatozoa were used in seminal plasma for artificial insemination, rapid sperm transport to the oviducts was observed in every animal examined $15 \mathrm{~min}$ after insemination. Rapid transport never occurred when non-motile spermatozoa were suspended in artificial media, but when motile spermatozoa were suspended in artificial media they were frequently recovered from the oviducts within $15 \mathrm{~min}$. Two successive artificial inseminations $15 \mathrm{~min}$ apart were each followed by rapid transport when both inseminates contained seminal plasma; when the second inseminate consisted of spermatozoa suspended in saline, only the first sperm population reached the oviducts. Rapid sperm transport was effectively blocked with the $\alpha$ adrenergic antagonist phenoxybenzamine. We therefore conclude that: (1) sperm motility is not required for rapid sperm transport in rabbits, (2) constituents of the seminal plasma may initiate rapid transport by stimulation of vaginal contractions, (3) independent contractions of the uterus under control of the sympathetic nervous system may continue the transport process once the spermatozoa reach the uterus.
\end{abstract}

\section{Introduction}

Immediately following insemination, rabbit spermatozoa are transported rapidly through the female reproductive tract to the upper oviduct and peritoneal cavity (Overstreet \& Cooper, 1978). Similar rapid transport phenomena occur in animals of many other mammalian species (Overstreet \& Katz, 1977; Overstreet, 1983). Since this speed of sperm passage far exceeds the swimming speed of the spermatozoon, it has been assumed that muscular contractions of the female viscera are primarily responsible for this event. Rapid transport of dead spermatozoa has been demonstrated in pigs (First, Short, Peters \& Stratman, 1968) and cows (VanDemark \& Moeller, 1951), but the role of sperm motility in the rapid transport of rabbit spermatozoa has been less clear since Dandekar, Vaidya \& Morris (1972) failed to recover rabbit spermatozoa from the oviducts when non-motile spermatozoa were inseminated.

The triggering event for rapid sperm transport has never been identified in any species. Previously discussed possibilities have included behavioural stimuli (Fuchs, 1972), mechanical stretching of the vagina (Fuchs, Olsen \& Petersen, 1965) and chemical stimulation by seminal plasma (Freund, 1973). We have therefore designed experiments to examine the role of sperm motility and female visceral contractions in the phase of rapid sperm transport in rabbits.

\section{Materials and Methods}

A total of 48 New Zealand White females were studied. Virgin and parous does were used, and all were tested for behavioural oestrus in the presence of a buck, which was allowed to mount but not to 
mate. Approximately $2 \mathrm{~h}$ later, artificial insemination was carried out. Semen was collected with an artificial vagina from New Zealand White male rabbits of proven fertility. Intravaginal artificial insemination (0.5-1.25 ml) was carried out by the method of Adams (1962).

Females were killed 15 min after artificial insemination by an intravenous overdose of pentobarbitone sodium (Nembutal: Abbott Laboratories, North Chicago, Illinois). The reproductive tract was exposed and divided with haemostat clamps into cranial vagina, cervices, uterine horns, upper and lower oviducal isthmi and upper and lower oviducal ampullae (Overstreet \& Cooper, 1978). Each region was flushed with $0.5 \%$ sodium dodecyl sulphate (SDS: Sigma Chemical Co., St Louis, Missouri) in distilled water. Each oviducal segment was flushed with $0.5 \mathrm{ml}$; the uteri and cervices as well as the surface of each ovary and each oviducal fimbria were each flushed with $1 \mathrm{ml}$ of the SDS solution. The number of spermatozoa on the ovarian surface and within each anatomical region was counted directly after centrifugation $(9380 \mathrm{~g})$ of the flushings for $1 \mathrm{~min}$ in a Beckman 152 Microfuge. When large numbers of spermatozoa were present, sperm concentrations in the cervix and uterus were calculated from multiple haemocytometer counts after suitable dilution. Details of the counting and collection procedures have been published previously (Overstreet \& Cooper, 1978).

\section{Experiment 1}

In 6 replicate experiments, spermatozoa were killed by exposure to the spermicide $+/-1-[2-$ (2,4-dichlorobenzyloxy)-n-octyl] imidazole oxalate, which was prepared in saline $(0.9 \%(\mathrm{w} / \mathrm{v})$ $\mathrm{NaCl}$ ) at a concentration of $350 \mu \mathrm{g} / \mathrm{ml}$. Spermatozoa were initially separated from seminal plasma by centrifugation, and the pellet was resuspended in $1 \mathrm{ml}$ spermicide solution. After 10 min the suspension was centrifuged at $500 \mathrm{~g}$ through a $5 \mathrm{ml}$ column of $10 \%$ ficoll (Ficoll, type 400, Sigma) in saline and the pellet was resuspended in the original seminal plasma. This procedure resulted in $100 \%$ immobilization of the sperm cells and visible disruption of the sperm head membranes. The suspension of dead spermatozoa in seminal plasma was then used for insemination. For comparison a control group of 4 females was similarly examined after artificial insemination with 1 $\mathrm{ml}$ untreated whole semen.

\section{Experiment 2}

Spermatozoa were killed by exposure to spermicide (Exp. 2A) as described in Exp. 1 (3 replicates) or by resuspension in distilled water followed by repeated freezing and thawing in liquid nitrogen (Exp. 2B, 3 replicates). After centrifugation, the sperm pellet was resuspended for artificial insemination in $1 \mathrm{ml}$ saline.

\section{Experiment 3}

In 2 replicate experiments (Exp. 3A) living spermatozoa were washed by centrifugation through $10 \%$ ficoll in saline and were resuspended in $1 \mathrm{ml}$ saline. In 5 other replicate experiments (Exp. 3B) the in-vitro fertilization medium of Brackett \& Oliphant (1975) was substituted for saline in the ficoll solution and in the inseminate.

\section{Experiment 4}

Spermatozoa were labelled with fluorescein isothiocyanate (FITC, Sigma) or tetramethyl rhodamine isothiocyanate (TRITC, Research Organics Inc., Cleveland, Ohio). These dyes have no effect on sperm motility or transport (Overstreet \& Bedford, 1974; Blazak, Overstreet, Katz \& Hanson, 1982), but because of their contrasting fluorescence (FITC, green; TRITC, red) they can be used to identify two different sperm populations after recovery from the female. The dyes were 
prepared in the Brackett \& Oliphant (1975) medium $(1.0 \mathrm{mg} \mathrm{FITC} / \mathrm{ml} ; 1.0 \mathrm{mg}$ TRITC/ml) as described previously (Blazak et al., 1982). One part of semen was diluted with 2 parts of the dye solution; after 10 min the labelled spermatozoa were washed through $10 \%$ ficoll in Brackett \& Oliphant's (1975) medium. Following centrifugation, the labelled sperm pellet was resuspended in seminal plasma or Brackett \& Oliphant's (1975) medium. In 8 replicate experiments both sperm populations were resuspended in seminal plasma (Exp. 4A). At $15 \mathrm{~min}$ after the first labelled population was used for insemination, the second population (with the contrasting label) was used. The tract was flushed $15 \mathrm{~min}$ after the second insemination, and the two populations were identified by phase-contrast fluorescence microscopy (Blazak et al., 1982). In a second series of 7 replicate experiments (Exp. 4B), only the first inseminate was suspended in seminal plasma; the second inseminate was suspended in Brackett \& Oliphant's (1975) medium. All other procedures were the same in the two sets of experiments.

\section{Experiment 5}

A group of 10 animals received an intravenous injection of the $\alpha$-adrenergic blocking agent phenoxybenzamine (Smith Kline and French Laboratories, Philadelphia, Pennsylvania) 20 min before artificial insemination with $1 \mathrm{ml}$ untreated semen. In 5 replicates a dosage of $1 \mathrm{mg} / \mathrm{kg}$ was given (Exp. 5A) and in 5 replicates a dosage of $3 \mathrm{mg} / \mathrm{kg}$ was adminstered (Exp. 5B).

\section{Results}

\section{Experiment 1 : transport of non-motile spermatozoa}

When non-motile spermatozoa were suspended in seminal plasma and the female tract was flushed $15 \mathrm{~min}$ after insemination, spermatozoa were recovered from the oviducts and ovaries of all 6 females (Table 1). Thousands of spermatozoa were present in the cervices of these animals but

Table 1. Number of spermatozoa recovered from the female rabbit reproductive tract at 15 min after artificial insemination

\begin{tabular}{|c|c|c|c|c|c|c|c|c|}
\hline \multirow{2}{*}{ Group } & \multirow{2}{*}{$\begin{array}{l}\text { No. of } \\
\text { animals }\end{array}$} & \multicolumn{7}{|c|}{ Median number of spermatozoa (range) } \\
\hline & & Inseminate & Cervix & Uterus & Isthmus & Ampulla & Fimbria & Ovary \\
\hline Control & 4 & $\begin{array}{c}4.8 \times 10^{8} \\
\left(1.5 \times 10^{6}-8.3 \times 10^{8}\right)\end{array}$ & $\left(1.0 \times 10^{5}-5.3 \times 10^{6} \times 10^{6}\right)$ & $\begin{array}{c}5 \\
(0-31)\end{array}$ & $\begin{array}{c}0^{*} \\
(0-16)\end{array}$ & $\begin{array}{c}14 \\
(0-35)\end{array}$ & $\begin{array}{c}32 \\
(0-45)\end{array}$ & $\begin{array}{c}26 \\
(0-50)\end{array}$ \\
\hline Exp. 1 & 6 & $\left(4.2 \times 10^{7}-1.6 \times 10^{8} \times 10^{8}\right)$ & $\begin{array}{c}7 \cdot 3 \times 10^{4} \\
\left(4.7 \times 10^{4}-1.5 \times 10^{5}\right)\end{array}$ & $\begin{array}{c}16 \\
(0-85)\end{array}$ & $\begin{array}{c}0^{*} \\
(0-851)\end{array}$ & $\begin{array}{c}29 \\
(25-42)\end{array}$ & $\begin{array}{c}42 \\
(29-300)\end{array}$ & $\begin{array}{c}112 \\
(30-140)\end{array}$ \\
\hline Exp. $2 A$ & 3 & $\begin{array}{c}9.2 \times 10^{7} \\
\left(1.6 \times 10^{7}-5.6 \times 10^{8}\right)\end{array}$ & $\begin{array}{c}0^{*} \\
(0-2)\end{array}$ & 0 & 0 & 0 & 0 & 0 \\
\hline Exp. 2B & 3 & $\begin{array}{l}6.8 \times 10^{8} \\
\left(4.9 \times 10^{8}-6.8 \times 10^{8}\right)\end{array}$ & 0 & 0 & 0 & 0 & 0 & 0 \\
\hline Exp. 3A & 2 & $6.8 \times 10^{7}-4.6 \times 10^{8}$ & $1.2 \times 10^{6}-7.9 \times 10^{6}$ & $0-4$ & 0 & $4-12$ & $6-40$ & $0-36$ \\
\hline Exp. 3B & 5 & 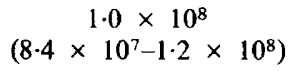 & $\left(6.0 \times 10^{5}-4.9 \times 10^{6} \times 10^{6}\right)$ & $\begin{array}{c}3 \\
(0-4)\end{array}$ & 0 & $\begin{array}{c}0^{*} \\
(0-2)\end{array}$ & $\begin{array}{c}0^{*} \\
(0-1)\end{array}$ & $\begin{array}{c}0^{*} \\
(0-30)\end{array}$ \\
\hline Exp. 4A & 8 & - & $\begin{array}{c}7.0 \times 10^{6} \\
\left(1.6 \times 10^{1}-6.0 \times 10^{7}\right)\end{array}$ & $\begin{array}{c}0^{*} \\
(0-11)\end{array}$ & 0 & $\begin{array}{c}20 \\
(0-74)\end{array}$ & $\begin{array}{c}15 \\
(0-80)\end{array}$ & $\begin{array}{c}18 \\
(0-60)\end{array}$ \\
\hline Exp. 4B & 7 & - & $\left(6.6 \times 10^{1}-6.3 \times 10^{2}\right)$ & 0 & 0 & $\begin{array}{c}8 \\
(0-31)\end{array}$ & $\begin{array}{c}0^{*} \\
(0-48)\end{array}$ & $\begin{array}{c}3 \\
(0-20)\end{array}$ \\
\hline Exp. 5A & 5 & $\begin{array}{c}6.7 \times 10^{8} \\
\left(9.7 \times 10^{7}-8.4 \times 10^{8}\right)\end{array}$ & $\begin{array}{c}6.0 \times 10^{6} \\
\left(0-1.4 \times 10^{7}\right)\end{array}$ & $\begin{array}{c}1 \\
(0-508)\end{array}$ & $\begin{array}{c}0^{*} \\
(0-5)\end{array}$ & $\begin{array}{c}0^{*} \\
(0-2)\end{array}$ & $\begin{array}{c}0^{*} \\
(0-8)\end{array}$ & $\begin{array}{c}0^{*} \\
(0-4)\end{array}$ \\
\hline Exp. 5B & 5 & $\begin{array}{c}6.7 \times 10^{7} \\
\left(5.3 \times 10^{6}-8.9 \times 10^{8}\right)\end{array}$ & $\left(\begin{array}{c}4.8 \times 10^{4} \\
\left(5.7 \times 10^{2}-6.4 \times 10^{5}\right)\end{array}\right.$ & 0 & 0 & 0 & 0 & 0 \\
\hline
\end{tabular}


fewer spermatozoa were recovered from the oviducts and ovary. There was evidence of rapid sperm transport in $3 / 4$ control animals (i.e. spermatozoa were recovered from the upper oviduct and/or ovary). Sperm numbers recovered from the cervix were higher in the control group (Table 1), but sperm recovery from the oviduct was similar in the experimental and control animals. In this experiment and in the following experiments, similar results were obtained with virgin and parous does.

\section{Experiment 2: effect of seminal plasma}

When non-motile spermatozoa were suspended in saline there was no evidence of rapid sperm transport in any of the 6 females (Table 1). In every animal spermatozoa were present in the vagina, but spermatozoa were observed in the cervix in only 1 animal. The results were similar regardless of whether the spermatozoa were killed by chemical (Exp. 2A) or physical (Exp. 2B) trauma.

\section{Experiment 3: sperm transport in artificial media}

There was evidence of rapid transport of spermatozoa suspended in artificial medium (2/2 in Exp. 3A and 3/5 in Exp. 3B). The number of spermatozoa recovered from the tract was similar to that observed after insemination of untreated semen (control), and sperm numbers in the cervix were 1-2 orders of magnitude greater than when non-motile spermatozoa were used in seminal plasma (Exp. 1). Although the progressive motility before artificial insemination was enhanced when the spermatozoa were suspended in the culture medium (Exp. 3B) rather than in saline (Exp. $3 \mathrm{~A})$, this had no clear influence on sperm transport.

\section{Experiment 4 : effect of 2 inseminations}

When both inseminates were suspended in seminal plasma (Exp. 4A), spermatozoa from both suspensions were consistently recovered from the ovaries and/or oviducts (data not shown). When the second inseminate was suspended in saline (Exp. 4B), none of these spermatozoa reached the oviduct although spermatozoa from the first suspension were recovered from every animal. The cervical sperm population, which was composed of cells from both inseminates, was small in all of the animals of this latter group (Exp. 4B).

\section{Experiment 5: effect of adrenergic blocking agent}

When does were treated with $1 \mathrm{mg}$ phenoxybenzamine/kg before insemination, spermatozoa were recovered from one or more regions of the oviduct in all 5 animals examined (Exp. 5A). In 4 of 5 animals, sperm numbers in the cervix were similar to those in the untreated animals inseminated with whole semen (no cervical spermatozoa were recovered from one animal). When the dose of phenoxybenzamine was increased to $3 \mathrm{mg} / \mathrm{kg}$, rapid sperm transport was blocked in every animal and sperm numbers were consistently low in the cervix (Exp. 5B). In every animal, numerous motile spermatozoa were present in the vagina at the time of the autopsy.

\section{Discussion}

These experiments demonstrate that sperm motility is not required for rapid sperm transport in rabbits. They also answer a fundamental criticism of the original rapid transport work, i.e. that the phenomenon could have been due to post-mortem contractions of the tract. Our observations also suggest that rapid transport may take place in at least two steps: (1) the passage of spermatozoa from the vagina to the uterus may occur passively by vaginal contraction under normal conditions, 
but the sperm cells have the ability to effect this passage by active swimming, and (2) co-ordinated uterine and oviducal contractions may then propel the spermatozoa to the upper oviduct and peritoneal cavity.

The initiator of sperm transport across the cervix may be one or more chemical constituents of the seminal plasma. Seminal plasma is known to contain a number of poorly characterized 'spasmogens' which can cause contraction of smooth muscle fibres of the female reproductive tract in vitro (Freund, 1973). Our observations suggest that seminal plasma could also exert such a localized action on the vaginal and/or cervical musculature in vivo. The resulting contractions may transport sperm cells into the cervical canal and perhaps as far as the uterine lumen.

Characteristic intrauterine pressure changes have been demonstrated at the time of mating in rabbits (Fuchs, 1972). There is no evidence that significant amounts of seminal plasma reach the rabbit uterus (Asch, Balmaceda \& Pauerstein, 1977) and it is likely that a set of independent stimuli initiate and control this activity. In our experiments rapid transport was demonstrated when there was no seminal plasma in the inseminate. However, whereas sperm motility was not required for rapid transport in the presence of seminal plasma, only motile spermatozoa were transported to the oviducts when seminal plasma was absent. This suggests that when passive transport was not induced by seminal plasma, the spermatozoa had to traverse the cervical canal by active swimming. The contractions of the rabbit uterus that are associated with mating continue for at least 5 min (Fuchs, 1972). This is a sufficient interval for rabbit spermatozoa to swim from the vagina to the uterus and then be transported rostrally before uterine contractions cease (e.g. a spermatozoon swimming at $40 \mu \mathrm{m} / \mathrm{sec}$ could traverse $12 \mathrm{~mm}$ in $5 \mathrm{~min}$ ). These interpretations are supported by the finding that, after insemination of non-motile spermatozoa in an artificial medium, virtually no spermatozoa were recovered from the cervix. In contrast, a significant cervical sperm population was present when there were actively motile spermatozoa and/or seminal plasma in the inseminate.

Successive artificial inseminations were each followed by rapid transport when both inseminates contained seminal plasma, but a second rapid transport was not observed when the second inseminate was suspended in saline. Fuchs (1972) observed two sets of uterine contractions when two successive matings were allowed, but the pressure tracings suggest that the second series of contractions was attenuated both in strength and duration. A shorter duration of uterine contraction may have contributed to the failure of rapid transport when the second inseminate contained no seminal plasma, i.e. uterine contractions may have ceased before the second sperm population could swim from the vagina to the uterus. The cervical sperm populations in this group of animals were among the smallest observed in this study. The reason for this is not clear, but it is possible that the second insemination 'washed out' a significant number of cells from the exocervix.

The observations of Fuchs (1972) suggested that the sympathetic nervous system was of principal importance in regulating intrauterine pressure changes and thus the early events of sperm transport. This notion is supported by our finding that rapid sperm transport was abolished by an $\alpha$ adrenergic blocking agent. Under conditions of natural mating uterine conditions may be initiated before intromission or simply by introduction of the male (Fuchs, 1972). Uterine contractions after artificial insemination could be initiated by pressure or stretch receptors in the vagina (Fuchs et al., 1965), although this mechanism was not investigated in our experiments.

The present experiments clearly demonstrate the pre-eminent role of female visceral contractions in rapid sperm transport. They also suggest an important function of the seminal plasma in initiating these events. Nevertheless, many questions about the process remain unanswered. The intriguing phenomenon of unilateral rapid transport in the left oviduct (Overstreet \& Cooper, 1978) was again apparent in the present experiments (for 30 of the 36 animals with rapid sperm transport, spermatozoa were recovered exclusively from the left side of the tract). The biological importance of rapid transport remains an enigma, particularly in the rabbit, a reflex ovulator in which rapid sperm transport appears to be lethal to the spermatozoa (Overstreet \& Cooper, 1978).

With the evolution of internal fertilization and limited numbers of oocytes, a highly controlled and complex physiological system must have emerged to ensure union of the gametes. If the 
physiology of this system is in any way analogous to other luminal transport systems such as that of the gastrointestinal tract, many local messengers may be involved in its control (Overstreet, 1983). In view of the fact that rapid sperm transport is apparently ubiquitous in mammals, this process may play a key role in signalling the onset of sperm transport to the female tract.

This research was supported by NIH Grant HD 11186. J.W.O. is the recipient of a NIH Research Career Development Award (HD 00224). The spermicide used in these studies was supplied by Dr Brian H. Vickery of Syntex Research, Palo Alto, California. We thank Dr Lani Johnson for her help in the execution and interpretation of the experiments.

\section{References}

Adams, C.E. (1962) Artificial insemination in rodents. In The Semen of Animals and Artificial Insemination, pp. 316-330. Ed. J. P. Maule. Commonwealth Agriculture Bureau, Farnham.

Asch, R.H., Balmaceda, J. \& Pauerstein, C.J. (1977) Failure of seminal plasma to enter the uterus and oviducts of the rabbit following artificial insemination. Fert. Steril. 28, 671-673.

Blazak, W.F., Overstreet, J.W., Katz, D.F. \& Hanson, F.W. (1982) A competitive in-vitro assay of human sperm fertilizing ability utilizing contrasting fluorescent sperm makers. J. Androl. 3, 165-171.

Brackett, B.G. \& Oliphant, G. (1975) Capacitation of rabbit spermatozoa in vitro. Biol. Reprod. 12, 260-274.

Dandekar, P., Vaidya, R. \& Morris, J. (1972) The effect of coitus on transport of sperm in the rabbit. Fert. Steril. 23, 759-762.

First, N.L., Short, R.E., Peters, J.B. \& Stratman, F.W. (1968) Transport and loss of boar spermatozoa in the reproductive tract of the sow. J. Anim. Sci. 27, 10371040 .

Freund, M. (1973) Mechanisms and problems of sperm transport. In The Regulation of Mammalian Reproduction, pp. 352-361. Eds S. J. Segal, R. Crozier, P. A. Corfman \& P. G. Condliffe. Charles C. Thomas, Springfield.
Fuchs, A.R. (1972) Uterine activity during and after mating in the rabbit. Fert. Steril. 23, 915-923.

Fuchs, A.R., Olsen, P. \& Petersen, K. (1965) Effect of distension of uterus and vagina on uterine motility and oxytocin release in puerperal rabbits. Acta endocr., Copenh. 50, 239-248.

Overstreet, J.W. (1983) Transport of gametes in the reproductive tract of the female mammal. In Mechanism and Control of Fertilization. Ed. J. F. Hartman. Academic Press, New York. (in press).

Overstreet, J.W. \& Bedford, J.M. (1974) Transport, capacitation and fertilizing ability of epididymal spermatozoa. J. exp. Zool. 189, 203-214.

Overstreet, J.W. \& Cooper, G.W. (1978) Sperm transport in the reproductive tract of the female rabbit. $I$. The rapid transit phase of transport. Biol. Reprod. 19, 101-114.

Overstreet, J.W. \& Katz, D.F. (1977) Sperm transport and selection in the female genital tract. In Development In Mammals, Vol. 2, pp. 31-65. Ed. M. H. Johnson. North-Holland Publishing Company, Amsterdam.

VanDemark, N.L. \& Moeller, A.N. (1951) Speed of spermatozoan transport in reproductive tract of estrous cow. Am. J. Physiol. 165, 674-679.

Received 25 February 1982 